Cahiers $d u$ MONDE RUSSE

\section{Cahiers du monde russe}

Russie - Empire russe - Union soviétique et États indépendants

$49 / 4 \mid 2008$

Destins individuels et terreur. Jeunesse dans la société post-stalinienne

\title{
Eric C. Landis, Bandits and Partisans
}

\section{Alexandre Sumpf}

\section{(2) OpenEdition}

Journals

Édition électronique

URL : https://journals.openedition.org/monderusse/6937

DOl : 10.4000/monderusse.6937

ISSN : $1777-5388$

Éditeur

Éditions de l'EHESS

Édition imprimée

Date de publication : 28 décembre 2008

Pagination : 736-738

ISBN : 978-2-7132-2197-2

ISSN : $1252-6576$

Référence électronique

Alexandre Sumpf, "Eric C. Landis, Bandits and Partisans », Cahiers du monde russe [En ligne], 49/4 I 2008, mis en ligne le 23 décembre 2009, consulté le 02 septembre 2022. URL : http://

journals.openedition.org/monderusse/6937 ; DOI : https://doi.org/10.4000/monderusse.6937

Ce document a été généré automatiquement le 2 septembre 2022

Tous droits réservés 


\title{
Eric C. Landis, Bandits and Partisans
}

\author{
Alexandre Sumpf
}

\section{RÉFÉRENCE}

Eric C. LANDIS, Bandits and Partisans. The Antonov Movement in the Russian Civil

War. Pittsburgh : University of Pittsburgh Press, 2008, 382 p.

1 Parmi les épisodes les plus symboliques de la guerre civile en Russie, l'insurrection de la province de Tambov, menée par Aleksandr Stepanovič Antonov, a déjà suscité nombre de travaux, principalement en Russie/URSS, où ont surtout été publiés des documents. Mais, pas plus que l'intervention alliée ou celle de la Légion tchèque (sans parler des légions hongroise ou italienne), elle n'avait fait l'objet d'une véritable synthèse. Celle qui est présentée ici est remarquable tant par l'ampleur de l'information que par la méthode d'approche. L'ouvrage d'Eric C. Landis frappe par la variété des types de documents convoqués, le juste choix opéré, le souci de faire la part des rumeurs, de contextualiser les témoignages, de dénoncer les effets de source et les inévitables biais. La narration, précise, laisse souvent l'interprétation ouverte, se refusant à l'exercice imposé de l'apposition d'une grille de lecture plaquée, confortable ou provocante, parfois stérilisante. L'alternance des regards des différents protagonistes rend assez bien compte des perceptions intriquées de l'époque, évitant l'écueil d'un «mystère Antonov » qui pourrait facilement faire mythe et l'illusion du recours aux « voix paysannes ». Leur mise en situation par l'auteur donne à comprendre que ces dernièresne forment pas un chœur homogène et représentent avant tout des « réponses ».

2 L'ouvrage, très documenté ( 80 pages de notes), se développe en neuf étapes répondant chacune à une question cruciale. La première fait le point sur les démobilisations, remobilisations et désertions de 1918 à 1920. La deuxième retrace brièvement l'itinéraire d'Antonov : « expropriateur » socialiste-révolutionnaire en 1905-1907, milicien local du nouveau régime pendant l'été 1917. Antonov a toujours revendiqué son expérience révolutionnaire, même si le lien avec le Parti socialiste-révolutionnaire (PSR)reste lâche, avant tout personnel et limité à l'échelle locale, rien ne permettant de dire que ce parti a 
commandé l'insurrection (ch. III). Il fonde son mouvement sur sa milice, devenue amicale combattante (družina), soutenant grâce à cette troupe et à ses liens politiques la révolte initiale contre la réquisition (razzverstka) qui embrase le 24 août 1920 la bourgade de Kamenka. Dans le sud-est de la province, les autorités se révèlent dès lors incapables de contrer les actions d'Antonov, puis de l'Armée partisane créée le 14 novembre 1920, au moment où on prétend que les «bandits" sont écrasés (ch. IV). De fait, les insurgés règnent sur un tiers de la province, ayant établi un maillage serré de "Conseils de la paysannerie laborieuse» (Sovety trudjaščegosja krest'janstva - STK) qui recrutent les troupes et tiennent l'économie pour garantir un approvisionnement minimal aux quatre armées du mouvement (ch. V).

Dès février 1920, dans une lettre aux autorités provinciales, Antonov affirmait lutter au nom de la démocratie et des classes laborieuses, et plus seulement de la paysannerie de Tambov. Son manifeste en 18 points, diffusé à la fin de l'année, revendique comme objectif le renversement du pouvoir soviétique et le retour aux libertés de 1917. Or le centre provincial persiste à le traiter en simple bandit, non en partisan, comme ce sera le cas après février 1921. Cette erreur d'appréciation, ainsi que les déclarations récurrentes d'éradication qui tentent de faire accroire au pouvoir central que la situation est sous contrôle, vont faire perdurer militairement un mouvement qui parait toujours plus invincible. Antonov échoue toutefois à soulever les régions voisines de Saratov ou de Penza, et même le soutien éphémère des 5000 hommes de Kolesnikov, qui a fui Voronej et pourrait établir un lien avec le mythique Mahno, ne conduit à rien (ch. VI). La situation se retourne avec l'envoi du général Tuhačevskij et des renforts d'unités spécialisées. Début juin 1921, l'armée d'Antonov est dispersée par la rapidité et la puissance de feu des véhicules blindés engagés dans l'opération (ch. VII). Le 24 juin 1922, dans une scène digne d'un western, le leader lui-même est abattu par un ancien partisan (ch. IX).

4 Ce succès, qui aura été long à obtenir, est dû à la stratégie tant militaire que politique adoptée par Tuhačevskij. Il est intéressant de suivre le débat sur le nombre de soldats à engager (130000 au final), qui résulte d'une estimation très confuse du volume des armées partisanes. Ce n'est qu'au moment où il prend au sérieux l'étendue de l'organisation adverse que Tuhačevskij renonce à la stratégie d'encerclement pour l'occupation militaire du terrain et la poursuite mobile. En témoigne un édifiant organigramme tentant de mettre en évidence une structure hiérarchique assimilable aux standards soviétiques - alors que l'insurrection est plutôt fondée sur la division en zones strictes de responsabilités. Les derniers rebelles se réfugient dans les forêts, d'où ils sont peu à peu délogés grâce à l'emploi de gaz qui, plus vanté que systématique, a surtout servi à cimenter la confiance pour des soldats peu aguerris.La fin du conflit aura ainsi servi de terrain d'entraînement à la guerre moderne pour une armée nouvellement constituée, au retard technologique avéré.

5 Le pouvoir soviétique ne met autant de temps à «liquider» l'insurrection que faute d'avoir su offrir une alternative crédible aux chefs et aux simples partisans - en témoigne l'échec des vagues successives d'amnistie. Le pouvoir local, en ces années de pénurie et de famine redoutée, réside dans la maîtrise de l'organisation économique par des STK sachant alterner compromission des individus dans l'illégalité et engagement idéologique (meetings), contrainte psychologique et mobilisations ponctuelles des ressources. Au même moment, les autorités provinciales, embourbées dans le débat politique lié au $\mathrm{X}^{\mathrm{e}}$ Congrès, sont totalement déconnectées des réalités de la campagne. 
Leur mise à l'écart par la dictature d'état, incarnée par la nomination d'AntonovOvseenko en tant que plénipotentiaire et par la purge de la Tcheka locale, lance la lutte sans rémission (surovo i bespoščadno) annoncée par les ordres n $n^{\circ} 130$ et 171.

6 Par ailleurs, Tambov expérimente la fin de la réquisition des grains avant même le passage à la NEP, proclamé par Lenin en mars 1921. Dans le plus captivant des chapitres (VIII), Landis dévoile les ressorts de la contre-mobilisation, menée au travers des comités révolutionnaires (revkomy) qui distribuent terres et semences, des serments individuels de repentis et des proclamations collectives, de la formation de milices d'autodéfense. On est frappé par la capacité d'anticipation des nouvelles mesures dont fait preuve, comme souvent, la paysannerie - moins hésitante à les interpréter dans le sens qui lui est le plus favorable. Craignant le retour au communisme de guerre, celle-ci souhaite voir enfin partir les 130000 bouches supplémentaires que constituent les forces d'occupation.

7 Tout au long de l'ouvrage sont soulignées avec justesse, dans la lignée des travaux de Peter Gatrell et Nick Baron, la porosité et la mixité de statuts sociaux en constant bouleversement. La récidive de la désertion en 1918, 1919 et 1920 est si bien mise en évidence que l'on aurait souhaité que cette réflexion incisive soit étendue à l'année 1917, en débat permanent, permettant ainsi, peut-être, de trancher entre les 200000 soldats de réserve désignés par Allen $\mathrm{K}$. Wildman et les 2 millions de soldats du front recensés par Joshua A. Sanborn'1 Le passage sur l'évolution de la population des camps de concentration à Tambov, la preuve fournie par l'auteur du réemploi des «fonctionnaires » des soviets de 1917 dans les STK comme dans les revkomy, plaident aussi en faveur d'une histoire fine des équilibres locaux du pouvoir au village, encore à écrire.

Enfin se dessine avec netteté la question cruciale de la démobilisation et du retour des anciens combattants, de leur réemploi dans l'administration soviétique à défaut de leur réadhésion au parti, choix configuré par leur rapport difficile à une communauté locale qui a rarement pris à cœur leurs intérêts quand il s'est agi de redistribuer les terres ou de protéger leurs familles des différents outsiders intervenant brutalement au village. On aurait aimé ici suivre certains parcours de ce type d'individus charnière, qui auraient permis de mieux dessiner les contours ambigus de l'expérience de la guerre civile, matricielle pour les bolcheviks engagés en 1917-1918, mais plus complexe à revendiquer par leurs camarades plus jeunes, au cœur du fossé générationnel.

\section{NOTES}

1. La référence de A. K. Wildman est son ouvrage The End of the Russian Imperial Army, tome 2 : The Road to Sovet Power and Peace, Princeton: Princeton Univ. Press, 1987, p. 366. La référence de J. Sanborn est sa communication «Le refus des soldats russes en 1917 » au colloque « Dans la guerre : accepter, endurer, refuser », Historial de la Grande Guerre, Péronne, 7-9 novembre 2008. 